\title{
Effect of the COVID-19 pandemic and the lockdown measures on the local stroke network
}

\author{
Valerio Brunetti ${ }^{1}$ - Aldobrando Broccolini ${ }^{1,2}$. Pietro Caliandro ${ }^{1}$. Riccardo Di lorio ${ }^{1}$ - Mauro Monforte ${ }^{1}$. \\ Roberta Morosetti ${ }^{1}$ - Carla Piano ${ }^{1}$ - Fabio Pilato ${ }^{1}$ - Simone Bellavia ${ }^{2}$ - Jessica Marotta ${ }^{2}$ Irene Scala $^{2}$. \\ Alessandro Pedicelli ${ }^{2,3}$. Mariano Alberto Pennisi ${ }^{2,4}$. Anselmo Caricato ${ }^{2,4}$. Cinzia Roberti ${ }^{5}$. Maria Concetta Altavista ${ }^{5}$. \\ Alessandro Valenza ${ }^{6}$. Marisa Distefano ${ }^{6}$. Emanuela Cecconi ${ }^{6}$ - Martina Fanella ${ }^{7}$. Sabina Roncacci ${ }^{7} \cdot$ Miriam Tasillo $^{7}$. \\ Paolo Calabresi $^{1,2} \cdot$ Giovanni Frisullo $^{1,8,9}$ (D) Giacomo Della Marca $^{1,2}$
}

Received: 30 September 2020 / Accepted: 5 January 2021 / Published online: 15 January 2021

(C) Fondazione Società Italiana di Neurologia 2021

\begin{abstract}
Introduction The COVID-19 outbreak highly impacted the acute ischemic stroke care management. The primary end point of the study was to evaluate the impact of the COVID-19 outbreak and the following lockdown measures on our hub-and-spoke network; the secondary end point was to evaluate if the impact of the COVID-19 outbreak was different in hub-and-spoke centers.

Methods This was a retrospective multicenter observational study conducted at the Stroke Units of Policlinico Gemelli, Ospedale San Filippo Neri, Ospedale di Belcolle, and Ospedale San Camillo de Lellis. We collected clinical reports of all consecutive patients admitted with diagnosis of acute ischemic stroke or transient ischemic attack (TIA) during the phase 1 of the lockdown period (11 March 2020-4 May 2020). As controls, we used all consecutive patients admitted for acute ischemic stroke or TIA in the same period of the previous year.

Results A total of 156 and 142 clinical reports were collected in 2019 and 2020, respectively. During the COVID-19 outbreak, we observed a reduction of number of thrombolysis, a reduction of the length of hospitalization, and an increase of pneumonia. Regarding performance indicators, we observed an increase in onset-to-door time and in door-to-groin time. We did not observe any statistically significant interaction between year (2019 vs 2020) and facility of admission (hub vs spoke) on all variables analyzed.

Discussion Our observational study, involving hub-and-spoke stroke network of a wide regional area, indicates that the COVID19 outbreak impacted on the acute stroke management. This impact was equally observed in hub as well as in spoke centers.
\end{abstract}

Keywords COVID-19 $\cdot$ Stroke $\cdot$ Cerebrovascular disease $\cdot$ Hub $\cdot$ Spoke

Giovanni Frisullo and Giacomo Della Marca share senior authorship.

Giovanni Frisullo

giovanni.frisullo@policlinicogemelli.it

1 Dipartimento Scienze dell'Invecchiamento, Neurologiche, Ortopediche e della Testa-Collo, Fondazione Policlinico Universitario A. Gemelli IRCCS - UOC Neurologia, Rome, Italy

2 Università Cattolica del Sacro Cuore, Largo Francesco Vito, 1, 00168 Rome, Italy

3 Dipartimento Diagnostica per Immagini, Radioterapia, Oncologia ed Ematologia, Fondazione Policlinico Universitario A. Gemelli IRCCS - UOC Radiologia e Neuroradiologia, Rome, Italy
4 Dipartimento Scienze dell'emergenza, anestesiologiche e della rianimazione, Fondazione Policlinico Universitario A. Gemelli IRCCS - UOC Anestesia, Rianimazione, Terapia Intensiva e Tossicologia Clinica, Rome, Italy

5 ASL Roma 1, San Filippo Neri Hospital - UOC Neurologia, Rome, Italy

6 Ospedale Belcolle - UOC Neurologia, Viterbo, Italy

7 Unità di Trattamento Neurovascolare (UTN) - Stroke Unit, Ospedale S. Camillo de Lellis, Rieti, Italy

8 Institute of Neurology, Catholic University, Rome, Italy

9 Policlinico Universitario “A. Gemelli”, Largo A. Gemelli, 8, 00168 Rome, Italy 


\section{Introduction}

Acute ischemic stroke (AIS) is a model of time-dependent disease since the aim of treatment is a highly prompt, safe, and effective arterial recanalization to restore perfusion of the ischemic brain tissue [1]. In order to ensure a fair distribution of care and to guarantee a high-quality standard of treatment, different models were proposed in the pre-hospital management of AIS [2]. The hub-and-spoke is one of the possible models adopted. In this setting, an anchor hospital (hub) offers a full array of services, complemented by secondary hospitals (spokes) which offer more limited service arrays. According to this model, the spoke ensures thrombolytic treatment $24 \mathrm{~h}$ a day while the mechanical thrombectomy treatment is carried out in the hub according to a drip-and-ship model [3].

The COVID-19 outbreak has had a different impact on the in-hospital and pre-hospital performance indicators of AIS care pathway [4]. Various experiences have been reported which describes the impact of the COVID-19 pandemic on the AIS management. The results of these observations are highly heterogeneous and probably reflect the model of AIS management adopted in each center [5]. In a previous report, we described the effect of the lockdown in our center which is a hub of the stroke network of the Lazio Region [6]. As follows, we evaluated the impact of the COVID-19 outbreak and the following lockdown measures on the hub-and-spoke network, by assessing pre-hospital and in-hospital care indicators. The primary end point of the study was to evaluate the impact of the COVID-19 outbreak and the following lockdown measures on our hub-and-spoke network; the secondary end point was to evaluate if the impact of the COVID-19 outbreak was different in hub-and-spoke centers, by measuring the interaction between year and facility of admission.

\section{Methods}

\section{Study designs and settings}

This retrospective multicenter observational study has been conducted at the Stroke Unit of Policlinico A. Gemelli in Rome (hub hospital) and at the Stroke Units of the three spokes of the regional stroke network (Presidio Ospedaliero San Filippo Neri, Rome; Ospedale di Belcolle, Viterbo; Ospedale San Camillo de Lellis, Rieti) serving the northern area of Lazio, with around 1.6 million inhabitants. One of the three spokes (Ospedale di Belcolle, Viterbo) is able to perform 24/7 mechanical thrombectomy independently. Moreover, due to the outbreak of COVID-19, the national healthcare system underwent a deep reorganization, and Policlinico A. Gemelli, Ospedale di Belcolle, and Presidio Ospedaliero San Filippo Neri were identified as COVID hospitals, and specific pathways were activated for patients with suspected or confirmed COVID-19 infection. Moreover, at the Presidio Ospedaliero San Filippo Neri, due to the logistic reorganization, neurological ward was displaced, and the number of beds in neurology unit was noticeably reduced.

In order to evaluate the impact of the lockdown measures due to the COVID-19 outbreak, we collected data in the phase 1 of the lockdown period (11 March 2020-4 May 2020), when measures to counter and contain the spread of SARSCoV-2 were valid throughout Italy.

This study was conducted according to the Declaration of Helsinki and approved by the Ethics Committee of Fondazione Policlinico Universitario Agostino Gemelli (Prot. 13729/20 ID:3065).

\section{Data sources, patients, and variables}

Data sources were clinical reports. We collected clinical reports of all consecutive patients admitted during the phase 1 of the lockdown period with diagnosis of AIS or transient ischemic attack (TIA) confirmed at the discharge (ICD-X codes 433, 434, 435, 436). As controls, we used all consecutive patients admitted for AIS and TIA in the same period of the previous year (11 March to 4 May 2019). The following variables were collected: total number of patients admitted in the local network, facility of admission (hub or spoke), age, gender, diagnosis (AIS or TIA), TOAST classification, other thrombotic manifestations, number of thrombolysis, number of thrombectomy, NIHSS at the onset, NIHSS at the discharge, stroke severity (mild, NIHSS 0-5; moderate, NIHSS 6-15; severe, NIHSS 16-42), modified Rankin scale at discharge, length of stay in hospital, fever, pneumonia, death, COVID-19 infection, onset-to-door time (ODT), door-to-CT time, length of stay in emergency room (ER), door-to-needle time (DNT), and door-to-groin time (DGT).

\section{Statistical analysis}

Continuous data were summarized using mean and standard deviation (SD) and median and interquartile range (IQR); categorical data were summarized using counts and percentages.

Statistical analysis was performed in multiple steps. In the first step, we compared the aforesaid variables between patients admitted during the phase 1 of the lockdown period (2020 group) and patients admitted during the same period of the previous year (2019 group). In order to verify the normality of the distribution of numerical variables of the samples, the Shapiro-Wilk test was performed; the threshold of significance was set at $p<0.05$. The distribution of variables was not normal; therefore, the Mann-Whitney $U$ test was used in the comparison of numerical variables, and Pearson's chisquare $\left(\chi^{2}\right)$ was used for non-numeric variables. The threshold 
for significance was $p<0.05$. Subsequently, to adjust the effect size of the variables for potential confounding effects, a multivariate logistic regression analysis was performed; the independent variable considered was the year $(2020$ vs 2019). The fitness of the multivariate model was tested by the Hosmer-Lemeshow (HL) test for logistic regression.

Finally, in order to test the effect of the interaction between COVID-19 and the facility of admission (time* group interaction), the comparison was performed by means of two-way analysis of variance (ANOVA). The independent variables considered were year (2019 vs 2020) and facility of admission (hub vs spoke). Due to the non-normal distribution of the variables, data normalization was performed before the ANOVA. A $p$ value of $<0.05$ was considered to reject the null hypothesis. We used the SPSS package (version 20) to perform statistical analysis.

\section{Results}

Between 11 March and 4 May, a total of 156 (74 men; age: mean $72.9 \pm 13.5$, median 75.6, IQR: 17.6 years) and $142(73$ men; age: mean $73.1 \pm 13.7$, median 75.0 , IQR: 15.3 years) clinical reports were collected in years 2019 and 2020, respectively. We observed a slight decrease in the number of AIS in our stroke hospitalization (156 in 2019 vs 142 in 2020, $9.0 \%$ ). This reduction involved both spokes (76 in 2019 vs 65 in 2020,-14.5\%) and hub (80 in 2019 vs 77 in 2020,$3.8 \%)$, but it was not statistically significant $\left(\chi^{2}=0.6113 ; p=\right.$ $0.26)$. The percentage of patients admitted at one of the Stroke Units of our network in years 2019 and 2020 was $76.2 \%$ and $73.1 \%$, respectively. The remaining percentage of patients were admitted at the neurology unit, internal medicine unit, or intensive care unit. In 2020, patients positive for SARSCoV-2 infection were admitted to dedicated COVID unit.

In 2020, we observed a significant reduction of number of thrombolysis (2019: $n=37$ vs 2020: $n=19 ; \chi^{2}=5.206 ; p=$ 0.023 ), reduction of the length of hospitalization (2019: mean $9.7 \pm 6.0$, median 8.2 , IQR: 6.9 days vs 2020 : mean $7.7 \pm 5.0$, median 6.4, IQR: 5.7 days; $U=12,802.5 ; p<0.001$ ), and a significant increase in cases of pneumonia (2019: $n=12 \mathrm{vs}$ 2020: $n=24 ; \chi^{2}=5.738 ; p=0.017$ ). No other significant differences were observed between 2019 and 2020 regarding clinical variables. Detailed results of demographic and clinical features of the study group are reported in Table 1; total number of patients admitted and number of thrombolysis and thrombectomy are reported in Fig. 1. Notably, in 2020, SARS-CoV-2 infections were diagnosed in nine patients (6.3\%) admitted in our stroke network; eight of them experienced symptoms consistent with COVID-19 with a median time from COVID-19 symptoms to the stroke of 4 days (IQR 10.5 days); one patient was asymptomatic. In only one case, stroke occurred in a patient admitted in COVID unit for bilateral interstitial pneumonia; in this case, stroke occurred 6 days after COVID-19 symptoms onset.

Regarding performance indicators, we observed a significant increase in ODT (2019: mean 485.5 \pm 903.8 , median 191.0, IQR: 364.0 vs 2020: mean $906.9 \pm 1563.3$, median 329.5, IQR: $586.5 \mathrm{~min} ; U=4663.0 ; p<0.001)$, a significant decrease of length of stay in ER (2019: mean 521.3 \pm 713.8 , median 231.5, IQR 364.8 min vs 2020: mean $263.7 \pm 320.2$, median 147.0, IQR $213.0 \mathrm{~min} ; p=0.003, U=10,780.0$ ), and a significant increase in DGT (2019: mean 104.2 \pm 51.1 , median 96.0, IQR 42.8 min vs 2020: mean $128.2 \pm 53.8$, median 120.5, IQR $19.3 \mathrm{~min} ; p=0.034, U=241.5$ ). No significant differences of DNT were observed (2019: mean $72.4 \pm 48.2$, median 59.5, IQR 25 min vs 2020: mean 59.0 \pm 20.6 , median 58.5 , IQR $19.5 \mathrm{~min} ; p=0.560$ ). Values of performance indicators are reported in Fig. 2. Results of univariate analysis were further confirmed by multivariate analysis. In particular, pneumonia and prolonged ODT occurred in year 2020, while prolonged length of stay in ER and of hospitalization occurred in 2019 (HL $p=0.139$ ). Detailed results of multivariate analysis are reported in Table 2 and in Fig. 3.

We did not observe any statistically significant interaction between year (2019 vs 2020) and facility of admission (hub vs spoke) on number of thrombolysis $(F(1,294)=0.341, p=$ $0.560)$, number of thrombectomy $(F(1,294)=0.262, p=$ $0.609)$, length of hospitalization $(F(1,286)=0.194, p=$ $0.660)$, ODT $(F(1,219)=0.404, p=0.526)$, DNT $(F(1$, $46)=0.537, p=0.467)$, DGT $(F(1,50)=1.067, p=0.307)$, NIHSS at the onset $(F(1,279)=0.003, p=0.959)$, pneumonia $(F(1,292)=0.689, p=0.407)$, and death $(F(1,291)=$ $0.047, p=0.829$ ) (for details, see Fig. 4). Detailed results of population characteristics classified according to year (2019 vs 2020) and facility of admission (hub vs spoke) are reported in Table 3.

\section{Discussion}

The COVID-19 pandemic has dramatically affected the entire health system mainly in patients with not communicable disorders [7]. Our observational study, involving a hub-andspoke stroke network of a wide Italian regional area, indicates that the COVID-19 outbreak can affect AIS management in this stroke care model.

Regarding the primary end point of our study, we observed a slight decrease in the frequency of AIS in our stroke network (156 in 2019 vs 142 in 2020,-9.0\%). This reduction was more evident in the spokes (76 in 2019 vs 65 in 2020, $14.5 \%$ ) compared to the hub (80 in 2019 vs 77 in 2020 , $3.8 \%)$. Nevertheless, the observed decrease did not reach statistical significance.

Regarding the most relevant performance indicators, we observed a reduction of total amount of thrombolysis 
Table 1 Demographic and clinical features: 2019 vs 2020 . A significant reduction of thrombolysis and of the length of hospitalization and an increase in the number of diagnosis of pneumonia were observed in 2020

\begin{tabular}{|c|c|c|c|c|c|c|c|c|c|}
\hline & \multicolumn{4}{|c|}{$2019(n=156)$} & \multicolumn{4}{|c|}{$2020(n=142)$} & \multirow[b]{2}{*}{$p$ value } \\
\hline & No. & $\%$ & Median & IQR & No. & $\%$ & Median & IQR & \\
\hline Facility of admission & & & & & & & & & 0.611 \\
\hline $\mathrm{Hub}$ & 80 & 51.3 & & & 77 & 54.2 & & & \\
\hline Spoke & 76 & 48.7 & & & 65 & 45.8 & & & \\
\hline Gender (M) & 74 & 47.4 & & & 73 & 51.4 & & & 0.493 \\
\hline Age (years) & & & 75.6 & 17.6 & & & 75.0 & 15.3 & 0.846 \\
\hline Diagnosis & & & & & & & & & 0.938 \\
\hline Stroke & 148 & 94.9 & & & 135 & 95.1 & & & \\
\hline TIA & 8 & 5.1 & & & 7 & 4.9 & & & \\
\hline TOAST classification* & & & & & & & & & 0.945 \\
\hline Large artery atherosclerosis & 28 & 20.9 & & & 31 & 25.4 & & & \\
\hline Cardio-embolism & 56 & 41.7 & & & 42 & 34.4 & & & \\
\hline Small vessel disease & 12 & 9.0 & & & 12 & 9.8 & & & \\
\hline $\begin{array}{l}\text { Stroke of other determined } \\
\text { etiologies }\end{array}$ & 6 & 4.5 & & & 2 & 1.6 & & & \\
\hline $\begin{array}{l}\text { Stroke of undetermined } \\
\text { etiology }\end{array}$ & 32 & 23.9 & & & 35 & 28.7 & & & \\
\hline $\begin{array}{l}\text { Other thrombotic } \\
\text { manifestations* }\end{array}$ & 5 & 3.7 & & & 5 & 4.1 & & & 0.880 \\
\hline Large artery occlusion* & 55 & 41.0 & & & 61 & 50.0 & & & 0.131 \\
\hline CT/MRI angiography* & 120 & 89.6 & & & 108 & 88.5 & & & 0.792 \\
\hline In-hospital stroke* & 5 & 3.7 & & & 3 & 2.5 & & & 0.559 \\
\hline Thrombolysis & 37 & 23.7 & & & 19 & 13.4 & & & 0.023 \\
\hline Thrombectomy & 27 & 17.3 & & & 29 & 20.4 & & & 0.492 \\
\hline NIHSS at the onset & & & 5.0 & 10.5 & & & 4.0 & 9.0 & 0.780 \\
\hline NIHSS at the discharge & & & 2.0 & 4.0 & & & 2.0 & 4.5 & 0.756 \\
\hline Stroke severity & & & & & & & & & 0.559 \\
\hline Mild (NIHSS 0-5) & 89 & 60.5 & & & 85 & 62.5 & & & \\
\hline Moderate (NIHSS 6-14) & 25 & 17.0 & & & 27 & 19.9 & & & \\
\hline Severe (NIHSS 15-42) & 33 & 22.4 & & & 24 & 17.6 & & & \\
\hline $\mathrm{mRS}$ at the discharge* & & & 2 & 3 & & & 2 & 3 & 0.787 \\
\hline $\begin{array}{l}\text { Length of stay in hospital } \\
\text { (days) }\end{array}$ & & & 8.2 & 6.9 & & & 6.4 & 5.7 & $<0.001$ \\
\hline Fever & 38 & 24.4 & & & 43 & 30.3 & & & 0.492 \\
\hline Pneumonia & 12 & 7.7 & & & 24 & 16.9 & & & 0.017 \\
\hline Death & 21 & 13.5 & & & 14 & 9.9 & & & 0.369 \\
\hline SARS-CoV-2 infection & & & & & 9 & 6.3 & & & \\
\hline $\begin{array}{l}\text { Time from COVID-19 symp- } \\
\text { toms to stroke (days) }\end{array}$ & & & & & & & 4 & 10.5 & \\
\hline
\end{tabular}

*Data from one of the spoke centers were not available $(n 2019=134 ; n 2020=122)$
[8-10]; on the other hand, the total number of thrombectomy performed in our network did not reduce. The reduction in the number of systemic thrombolysis could be due to several factors. In our opinion, it mainly reflects the increased onset-todoor time. Therefore, most of the patients arrived at our observation beyond the time window $(4.5 \mathrm{~h})$ for thrombolytic treatment. This finding may be explained by a delay in the patient calling an ambulance, or due to changes in dispatch during the pandemic or an overbooking in pre-hospital pathway. Other factors could have contributed to the reduction of the number of thrombolysis. First, the limited experience of healthcare practitioners using intravenous recombinant tissue plasminogen activator (rtPA) in settings of the SARS-CoV-2 infection could have influenced rates of administration. Second, healthcare professionals may have been concerned about possible CT scanner contamination before administering rtPA. Third, healthcare professionals may have faced difficulties in the evaluation and management of acute stroke 
Fig. 1 Total number of patients admitted, thrombolysis, and thrombectomy: 2019 vs 2020 . A significant reduction of total number of thrombolysis was observed in 2020 (19) compared to 2019 (37)

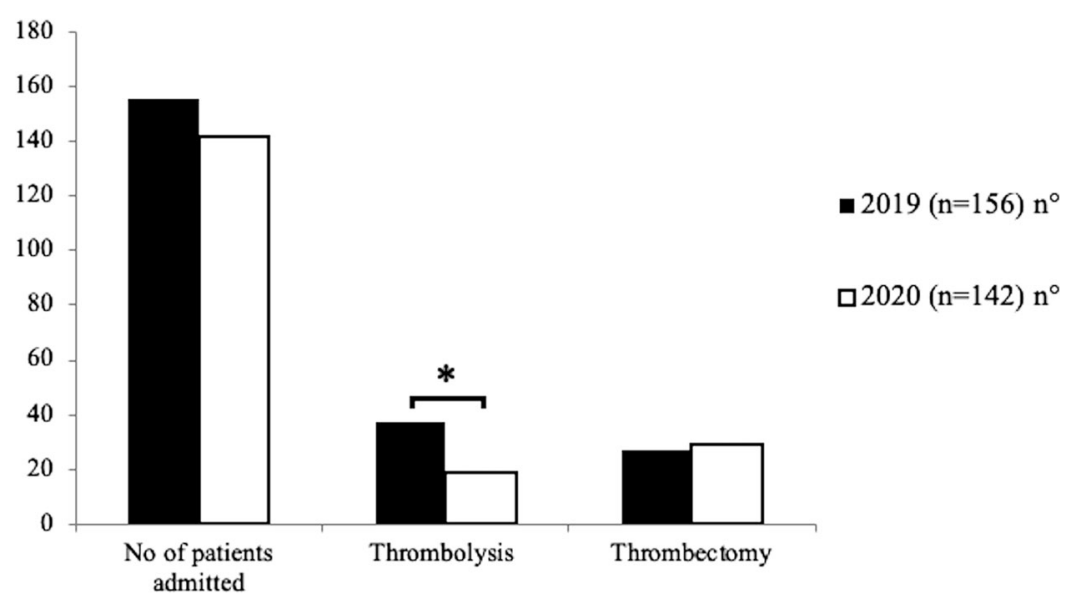

patients who were at risk of acquiring COVID-19 from suspected or confirmed COVID-19 patients who were asymptomatic during the prodromal period. Implementing specific in-hospital emergency and pre-hospital emergency management guidelines could aid in promoting the use of rtPA in the COVID-19 outbreak. Furthermore, we observed a

Onset to door(minutes)

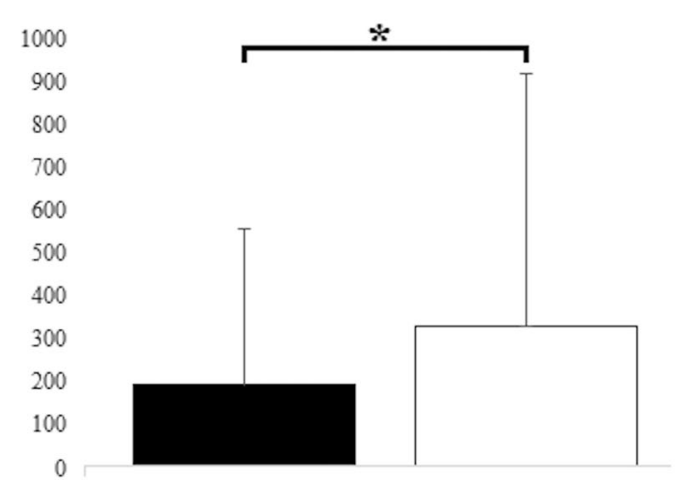

Door to needle (minutes)

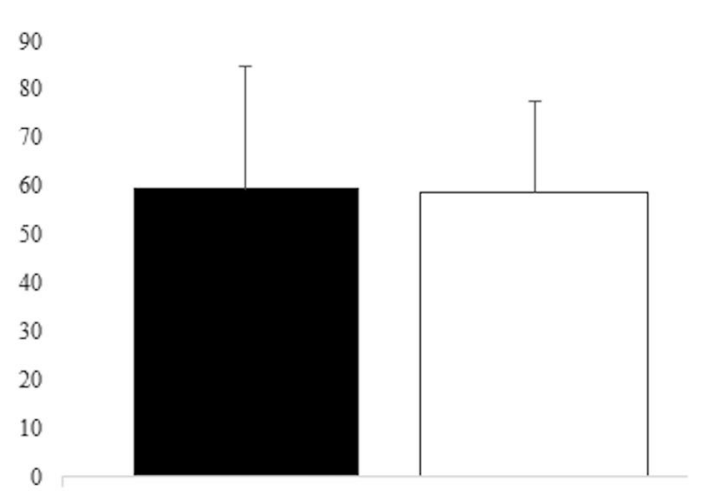

significant increase in DGT in 2020. This probably reflects a more complex management of stroke patients with large vessel occlusion, which in many cases required intensive care including orotracheal intubation and availability of intensive care monitoring. All intubated patients in our network were considered as suspected COVID-19 and therefore required

\section{Length of stay in ER (minutes)}
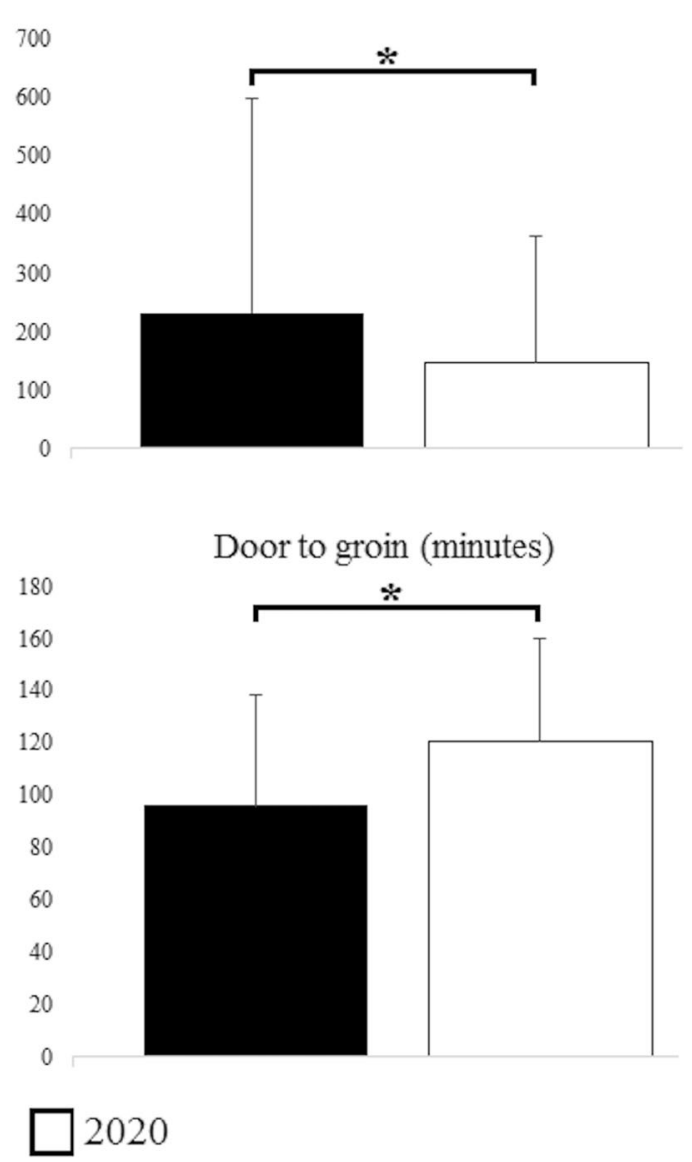

No significant modification of door-to-needle time was observed in 2020 compared to 2019
Fig. 2 Performance indicators of stroke network: 2019 vs 2020. Onsetto-door time and door-to-groin time were significantly longer in 2020 . Length of stay in emergency room (ER) was significantly shorter in 2020. 
Table 2 Results of multivariate analysis. Pneumonia and longer onsetto-door are prevalent in 2020, whereas longer hospitalization and longer length of stay in emergency room (ER) are prevalent in 2019

\begin{tabular}{llll}
\hline & Odds ratio & CI (95\%) & $p$ value \\
\hline Gender & 1.399 & $(0.679-2.886)$ & 0.363 \\
Age & 1.018 & $(0.989-1.047)$ & 0.232 \\
Length of hospitalization & 0.906 & $(0.831-0.987)$ & $\mathbf{0 . 0 2 3}$ \\
Length of stay in ER & 0.998 & $(0.997-0.999)$ & $\mathbf{0 . 0 0 8}$ \\
Onset-to-door time & 1.001 & $(1.000-1001)$ & $\mathbf{0 . 0 2 3}$ \\
Door-to-CT time & 1.000 & $(0.998-1.003)$ & 0.775 \\
Thrombolysis & 0.607 & $(0.241-1.526)$ & 0.289 \\
Thrombectomy & 2.254 & $(0.697-7.295)$ & 0.175 \\
Fever & 2.661 & $(0.849-8.336)$ & 0.093 \\
Pneumonia & 5.803 & $(1.065-31.602)$ & $\mathbf{0 . 0 4 2}$ \\
NIHSS at onset & 0.930 & $(0.832-1.041)$ & 0.208 \\
NIHSS at discharge & 0.986 & $(0.892-1.088)$ & 0.774 \\
\hline
\end{tabular}

COVID-dedicated time-consuming procedures before undergoing endovascular treatment. These procedures included a COVID-specific triage, execution of oronasal swab, chest $\mathrm{CT}$ or X-ray, and wearing of personal protective equipment by all members of the interventional staff.

In the post-treatment phase of AIS management, we observed a significant reduction of the length of hospitalization and an increased rate in the diagnosis of pneumonias. The

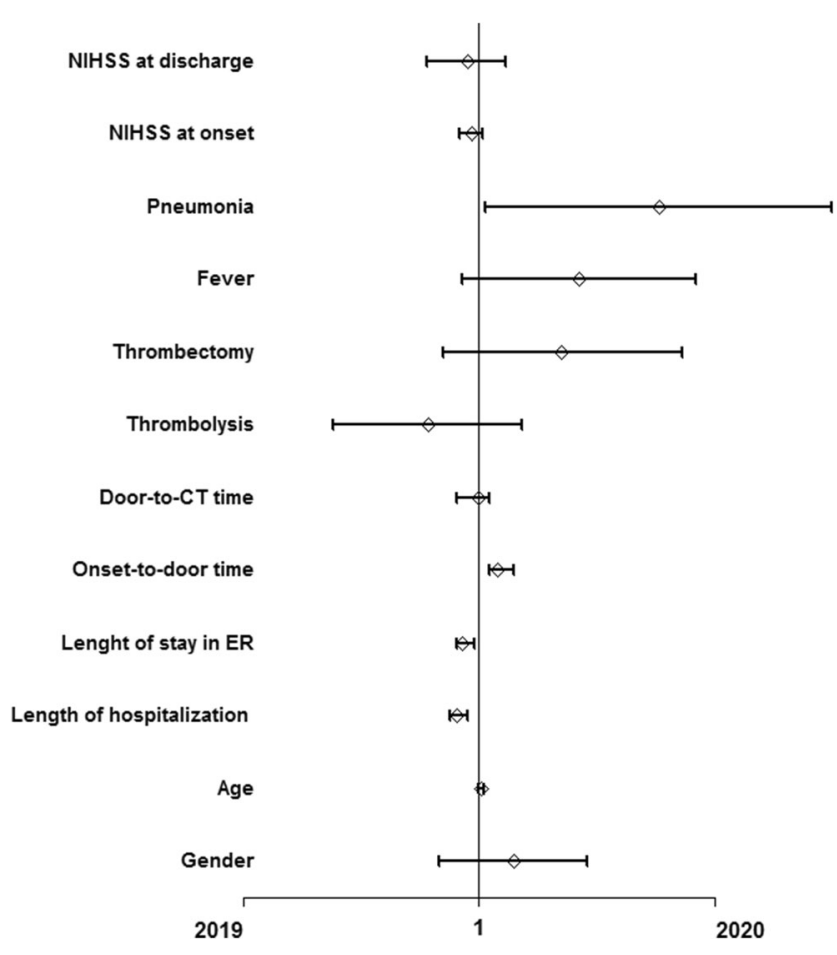

Fig. 3 Forest plot showing results of multivariate analysis: 2019 vs 2020. Pneumonia and prolonged onset-to-door occurred in year 2020, while prolonged length of stay in ER and of hospitalization occurred in 2019 shorter hospitalization reflects the need to reduce the exposure to SARS-CoV-2 and the shorter waiting lists for clinical and instrumental tests, due to the interruption of all non-urgent activities. The increased number of diagnosis of pneumonias is largely due to the systematic use of chest CT scan, rather than the less sensitive chest $\mathrm{X}$-ray, for the evaluation of respiratory symptoms in acute patients during the COVID-19 outbreak. Moreover, all patients of both periods (2019 and 2020) were systematically screened for swallowing with bedside test, and the prevalence of dysphagia did not differ between the two periods; therefore, the increased proportion of pneumonia in 2020 is not related to an increased prevalence of aspiration pneumonia.

We did not observe significant differences regarding clinical characteristics of patients admitted for stroke during the observation period compared to the previous year. In particular, no differences were observed regarding stroke severity, NIHSS at admission and at discharge, mRS at discharge, inhospital death, proportion of large artery vessel occlusion, and stroke subtype. These data suggest that stroke remains a primary emergency and, therefore, it is of paramount importance to guarantee the functionality of stroke services and to promote sensitization campaigns on time-dependent pathologies. Further studies aimed to investigate the long-term outcome in stroke patients during a pandemic will clarify the effect of the COVID-19 outbreak on prognosis of patients affected by acute cerebrovascular accidents.

The prevalence of SARS-CoV-2 infection in our population was $6.3 \%$, apparently higher to that one reported in the general population of the Lazio Region (1\%) where we operate [11]. This data could reflect a promoting role of SARSCoV-2 in the pathogenesis of stroke, as largely described in the recent literature [12]. On the other hand, it could be the result of the systematic screening for SARS-CoV-2 performed in all patients admitted to the hospitals.

To test the hypothesis that the lockdown measures differently affected the stroke management in the hub center versus spoke centers, we analyzed the effect of the interaction between time of observation (2019 vs 2020) and site of observation (hub vs spoke). This comparison did not show any significant modifications for any of the variables analyzed, suggesting that the lockdown measures had a similar impact on all the network nodes.

Experiences from other stroke centers can hardly be compared to what is observed in our network. First, the spread of the COVID-19 outbreak was largely heterogeneous in different countries and even in different regions of the same country; moreover, each country adopted a different model of organization. Therefore, the results reported in literature are not homogeneous and, often, contrasting. For example, most centers observed a reduction in the frequency of admission for AIS [13-16], while, concerning the indicators of stroke care, some centers did not report substantial modifications [17], 


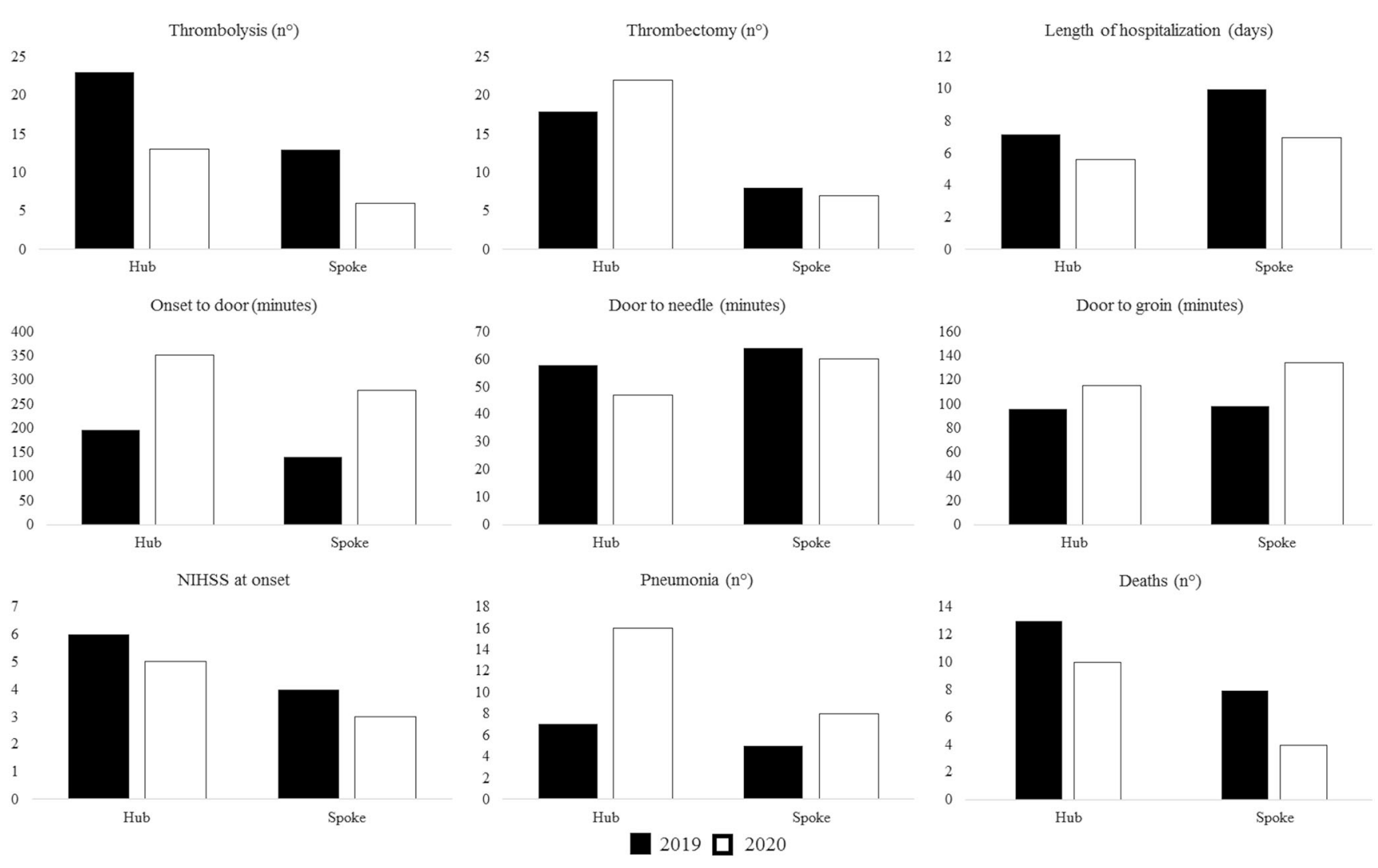

Fig. 4 Results of the interaction between time of observation (2019 vs 2020) and site of observation (hub vs spoke). No significant differences were observed for the variables analyzed

while others reported a negative impact of stroke care performances $[18,19]$.

During the outbreak, our regional hub-and-spoke network did not change conversely to other Italian experiences [20]. For example, in our network, before the admission to emergency department, all patients underwent a two-step triage. In the first step, the risk of COVID-19 infection was evaluated. Based on the pre-triage, the patients classified as "suspected COVID-19" entered a dedicated pathway, whereas the patients without any suspicion of infection moved on to the ordinary hospital triage. Interestingly, during the lockdown period, all non-urgent activities were suspended, and, in turn, we observed a shorter waiting list for radiological or laboratory tests for patients affected by stroke. Moreover, the strict collaboration with inpatient rehabilitation facilities allowed us to get a shorter length of hospitalization during the COVID-19 outbreak, reducing the risks of exposure to SARS-CoV-2. The latter point is of paramount importance, in order to guarantee to stroke patients a prompt physical therapy.

The main limitation of our study is the relatively short duration of the observation, which is, however, closely related to the first phase of COVID-19 breakdown, and the consequently relatively small number of patients included in the analysis. These results need to be confirmed in different hub-andspoke stroke networks to demonstrate the sustainability of this stroke care model during the COVID-19 pandemic. Our results could be affected by a selection bias due to the specific nature of our stroke care network and not including the full range of AIS patients. Therefore, it is important to closely monitor the changes in stroke rates during various phases of the pandemic because the impact of COVID-19 on the healthcare system will be sustained and long lasting.

In conclusion, our report indicates that the COVID-19 pandemic and the consequent lockdown measures have impacted stroke care in our stroke network and that this impact mainly involved pre-hospital management. In particular, intravenous thrombolysis was mainly affected due to an increase of ODT or maybe due to a delay in seeking treatment and fear of exposure to COVID-19 in hospitals or a change in dispatch during the pandemic or an overloading in pre-hospital pathway. This is an alarming finding for healthcare authorities, and specific in-hospital emergency and pre-hospital emergency management guidelines need to be implemented to promote the use of rtPA during the COVID-19 outbreak. Within the intra-hospital management, a delay was observed in procedures involving patients classified as suspected COVID-19. Despite the recent shift towards prioritization of COVID-19 care, it is equally important to ensure that nonCOVID-19 patients, such as stroke patients, continue to receive timely access to care and adequate support. Finally, in 
Table 3 Demographic features, clinical features, and performance indicators of patients admitted in hub and in spokes in 2019 and 2020

\begin{tabular}{|c|c|c|c|c|c|c|c|c|c|c|c|c|c|c|c|c|}
\hline & \multicolumn{8}{|c|}{$2019(n=156)$} & \multicolumn{8}{|c|}{$2020(n=142)$} \\
\hline & \multicolumn{4}{|c|}{ Hub $(n=80)$} & \multicolumn{4}{|c|}{ Spoke $(n=76)$} & \multicolumn{4}{|c|}{ Hub $(n=77)$} & \multicolumn{4}{|c|}{ Spoke $(n=65)$} \\
\hline & No. & $\%$ & Median & IQR & No. & $\%$ & Median & IQR & No. & $\%$ & Median & IQR & No. & $\%$ & Median & IQR \\
\hline Gender (M) & 41 & 51.3 & & & 33 & 43.4 & & & 41 & 53.2 & & & 32 & 49.2 & & \\
\hline Age (years) & & & 75.3 & 15.8 & & & 76.0 & 19.5 & & & 73.4 & 17.5 & & & 77.0 & 13.0 \\
\hline \multicolumn{17}{|l|}{ Diagnosis } \\
\hline Stroke & 75 & 93.8 & & & 73 & 96.1 & & & 72 & 93.5 & & & 63 & 96.9 & & \\
\hline TIA & 5 & 6.2 & & & 3 & 3.9 & & & 5 & 6.5 & & & 2 & 3.1 & & \\
\hline Thrombolysis & 24 & 30.0 & & & 13 & 17.1 & & & 13 & 16.9 & & & 6 & 9.2 & & \\
\hline Thrombectomy & 19 & 23.8 & & & 8 & 10.5 & & & 22 & 28.6 & & & 7 & 10.8 & & \\
\hline NIHSS at the onset & & & 6.0 & 14.0 & & & 4.0 & 6.0 & & & 5.0 & 9.3 & & & 3.0 & 7.3 \\
\hline NIHSS at the discharge & & & 2.0 & 4.0 & & & 2.0 & 4.0 & & & 2.0 & 5.0 & & & 1.0 & 3.5 \\
\hline Length of stay in hospital (days) & & & 7.2 & 7.3 & & & 10.0 & 5.3 & & & 5.6 & 6.2 & & & 7.0 & 6.0 \\
\hline Onset-to-door (min) & & & 197.0 & 331.5 & & & 140.0 & 407.0 & & & 350.5 & 670.3 & & & 279.0 & 496.0 \\
\hline Door-to-needle (min) & & & 58.0 & 22.0 & & & 64.0 & 30.0 & & & 47.0 & 18.0 & & & 60.0 & 37.0 \\
\hline Door-to-groin (min) & & & 96.0 & 40.3 & & & 98.5 & 38.8 & & & 115.0 & 33.0 & & & 134.5 & 53.5 \\
\hline Length of stay in ER (min) & & & 222.0 & 389.5 & & & 245.0 & 352.5 & & & 133.0 & 219.5 & & & 172.0 & 193.0 \\
\hline Fever & 16 & 20.0 & & & 22 & 28.9 & & & 25 & 32.5 & & & 18 & 27.7 & & \\
\hline Pneumonia & 7 & 8.8 & & & 5 & 6.6 & & & 16 & 20.8 & & & 8 & 12.3 & & \\
\hline Death & 13 & 16.3 & & & 8 & 10.5 & & & 10 & 13.0 & & & 4 & 6.2 & & \\
\hline COVID-19 infection & 0 & 0.0 & & & 0 & 0.0 & & & 4 & 5.2 & & & 5 & 7.7 & & \\
\hline
\end{tabular}

our case, the impact of the COVID-19 outbreak on AIS management was equally observed in hub as well as spoke centers.

\section{Compliance with ethical standards}

Conflict of interest The authors declare that they have no conflict of interest.

Ethical approval The study has been performed in accordance with the ethical standards laid down in the 1964 Declaration of Helsinki and its later amendments. This study was approved by the local Ethics Committee (Prot. 13729/20 ID:3065).

\section{References}

1. Saver JL, Smith EE, Fonarow GC, Reeves MJ, Zhao X, Olson DM, Schwamm LH, GWTG-Stroke Steering Committee and Investigators (2010) The "golden hour" and acute brain ischemia: presenting features and lytic therapy in $>30,000$ patients arriving within 60 minutes of stroke onset. Stroke 41(7):1431-1439

2. Fassbender K, Walter S, Grunwald IQ, Merzou F, Mathur S, Lesmeister M, Liu Y, Bertsch T, Grotta JC (2020) Prehospital stroke management in the thrombectomy era. Lancet Neurol 19(7):601-610

3. Bekelis K, Missios S, Coy S, Mayerson B, MacKenzie TA (2019) Emergency medical services for acute ischemic stroke: hub-andspoke model versus exclusive care in comprehensive centers. J Clin Neurosci 60:12-16
4. Frisullo G, De Belvis AG, Marca GD, Angioletti C, Calabresi P (2020) Stroke integrated care pathway during COVID-19 pandemic. Neurol Sci 41(7):1673-1675

5. Bersano A, Kraemer M, Touze E, Weber R, Alamowitch S, Sibon I et al (2020) Stroke care during the COVID-19 pandemic: experience from three large European countries. Eur J Neurol 27:1794 1800

6. Frisullo G, Brunetti V, Di Iorio R, Broccolini A, Caliandro P, Monforte $\mathrm{M}$ et al (2020) Effect of lockdown on the management of ischemic stroke: an Italian experience from a COVID hospital. Neurol Sci 41:2309-2313

7. Azarpazhooh MR, Morovatdar N, Avan A, Phan TG, Divani AA, Yassi N, Stranges S, Silver B, Biller J, Tokazebani Belasi M, Kazemi Neya S, Khorram B, Frydman A, Nilanont Y, Onorati E, di Napoli M (2020) COVID-19 pandemic and burden of noncommunicable diseases: an ecological study on data of 185 countries. J Stroke Cerebrovasc Dis 29(9):105089

8. Hsiao J, Sayles E, Antzoulatos E, Stanton RJ, Sucharew H, Broderick JP et al Effect of COVID-19 on emergent stroke care: a regional experience. Stroke 2020:STROKEAHA120030499

9. Pop R, Quenardelle V, Hasiu A, Mihoc D, Sellal F, Dugay MH, Lebedinsky PA, Schluck E, la Porta A, Courtois S, Gheoca R, Wolff V, Beaujeux R (2020) Impact of the COVID-19 outbreak on acute stroke pathways - insights from the Alsace region in France. Eur J Neurol 27:1783-1787

10. Tejada Meza H, Lambea Gil A, Sancho Saldana A, Villar Yus C, Pardinas Baron B, Sagarra Mur D et al (2019) Ischaemic stroke in the time of coronavirus disease. Eur J Neurol:2020

11. Istituto Nazionale di Statistica - Ministero della Salute. Primi risultati dell'indagine di sieroprevalenza sul SARS-CoV-2. 2020 
12. Bhatia R, Pedapati R, Komakula S, Srivastava MVP, Vishnubhatla S, Khurana D (2020) Stroke in coronavirus disease 2019: a systematic review. J Stroke 22(3):324-335

13. Markus HS, Brainin M (2020) COVID-19 and stroke-a global World Stroke Organization perspective. Int J Stroke 15(4):361-364

14. Kansagra AP, Goyal MS, Hamilton S, Albers GW (2020) Collateral effect of Covid-19 on stroke evaluation in the United States. N Engl J Med 383(4):400-401

15. Paliwal PR, Tan BYQ, Leow AST, Sibi S, Chor DWP, Chin AXY, Yau YW, Cross GB, Wong LYH, Chia MLJ, Quak Z, Chua CYK, Tang DKK, Zune ET, Hung J, Goh Y, Jing M, Gopinathan A, Yang C, Ahmad A, Khoo DXL, Lee CCM, Seet RCS, Sharma VK, Teoh HL, Yeo LLL, Chan BPL (2020) Impact of the COVID-19 pandemic on hyperacute stroke treatment: experience from a comprehensive stroke centre in Singapore. J Thromb Thrombolysis 50: 596-603

16. Sacco S, Ricci S, Ornello R, Eusebi P, Petraglia L, Toni D et al Reduced admissions for cerebrovascular events during COVID-19 outbreak in Italy. Stroke 2020:STROKEAHA120031293

17. Rinkel LA, Prick JCM, Slot RER, Sombroek NMA, Burggraaff J, Groot AE, Emmer BJ, Roos YBWEM, Brouwer MC, van den
Berg-Vos RM, Majoie CBLM, Beenen LFM, van de Beek D, Visser MC, van Schaik SM, Coutinho JM (2020) Impact of the COVID-19 outbreak on acute stroke care. J Neurol

18. Neves Briard J, Ducroux C, Jacquin G, Alesefir W, Boisseau W, Daneault N, Deschaintre Y, Eneling J, Gioia LC, Iancu D, Odier C, Raymond J, Roy D, Stapf C, Weill A, Poppe AY (2020) Early impact of the COVID-19 pandemic on acute stroke treatment delays. Can J Neurol Sci:1-15

19. McConachie D, McConachie N, White P, Crossley R, Izzath W (2020) Mechanical thrombectomy for acute ischaemic stroke during the COVID-19 pandemic: changes to UK practice and lessons learned. Clin Radiol 75:795.e7-795.e13

20. Candeloro E, Carimati F, Tabaee Damavandi P, Princiotta Cariddi L, Banfi P, Clemenzi A, Gallazzi M, Mauri M, Rebecchi V, Baruzzi F, Giorgianni A, Tozzi M, Bianchi M, Ageno W, Versino M (2020) An example of a stroke unit reshaping in the context of a regional hub and spoke system in the COVID-19 era. Front Neurol 11:1029

Publisher's note Springer Nature remains neutral with regard to jurisdictional claims in published maps and institutional affiliations. 